\title{
A New Mechatronic Assistance System for the Neurosurgical Operating Theatre: Implementation, Assessment of Accuracy and Application Concepts
}

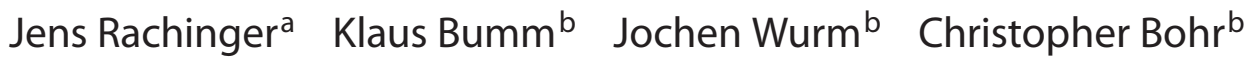

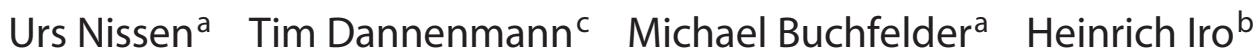 \\ Christopher Nimsky ${ }^{a}$
}

Departments of a Neurosurgery and b Otorhinolaryngology, Head and Neck Surgery, and

'Institute of Medical Physics, University of Erlangen-Nuremberg, Erlangen, Germany

\section{Key Words}

Neurosurgery · Robotics · Frameless stereotaxy, accuracy

\begin{abstract}
Objective: To introduce a new robotic system to the field of neurosurgery and report on a preliminary assessment of accuracy as well as on envisioned application concepts. Based on experience with another system (Evolution $1^{\circledR}$, URS Inc., Schwerin, Germany), technical advancements are discussed. Material/Methods: The basic module is an industrial 6 degrees of freedom robotic arm with a modified control element. The system combines frameless stereotaxy, robotics, and endoscopy. The robotic reproducibility error and the overall error were evaluated. For accuracy testing CT markers were placed on a cadaveric head and pinpointed with the robot's tool tip, both fully automated and telemanipulatory. Applicability in a clinical setting, user friendliness, safety and flexibility were assessed. Results: The new system is suitable for use in the neurosurgical operating theatre. Hard- and software are user-friendly and flexible. The mean reproducibility error was $0.052-0.062 \mathrm{~mm}$, the mean overall error was
\end{abstract}

J.R. and K.B. contributed equally to this work.

\section{KARGER}

Fax +41613061234 E-Mail karger@karger.ch www.karger.com
(C) 2007 S. Karger AG, Basel

1011-6125/07/0855-0249\$23.50/0

Accessible online at:

www.karger.com/sfn
$0.816 \mathrm{~mm}$. The system is less cumbersome and much easier to use than the Evolution 1. Conclusions: With its userfriendly interface and reliable safety features, its high application accuracy and flexibility, the new system is a versatile robotic platform for various neurosurgical applications. Adaptations for different applications are currently being realized.

Copyright $\odot 2007$ S. Karger AG, Basel

\section{Introduction}

Mechatronic systems, also known as robotic systems, are able to perform repetitive motions with an excellent geometric accuracy. They can process huge amounts of diverse information and do not fatigue [1]. To utilize these advantages for surgical procedures, industrial robotic systems have been adapted for use in different medical disciplines and some completely new systems have been developed. In general, there are supervisory controlled robotic systems in which the operation, or a certain step of it, is performed by the robotic system autonomously according to a plan elaborated by the surgeon. In this setting the surgeon merely works as a supervisor. In contrast to these are telemanipulatory systems in which

E-Mail jens.rachinger@medizin.uni-halle.de 
the surgeon controls each movement of the system directly via an on-line input device, e.g. a (force-feedback) joystick. This approach follows the master-slave principle with the surgeon in the role of the master. In a third variant, the shared control systems, the surgeon and robot share control of the surgical instrument with the surgeon remaining in control of the procedure itself and the robot providing steady-hand manipulation [2]. Meanwhile, robotic systems are used and evaluated in the fields of orthopedics [3], urology [4, 5], general surgery [6], and cardiac surgery [7]. But there is also great effort to implement this innovative technology in surgery around the head $[8,9]$. In neurosurgery, there are on one hand passive-robotic-assisted procedures in which the robot is guided by the surgeon's hand representing a telemanipulatory approach (telesurgical systems). In this way such systems can help with endoscopic 3rd ventriculocisternostomy [10] and with the placement of pedicle screws in spine surgery. Furthermore, they may enable guidance of the endoscope in transsphenoidal and other skull-base surgeries [11] and assist in brain biopsies, removal of brain tumors [12] and implantation of electrodes for deep brain stimulation [13]. On the other hand there are active-robotic-supported procedures in which certain steps of the operation are actively executed by the robot (supervisory controlled robotic systems). The robot could work here as an active endoscope-holding device or perform biopsies as well as drill and insert pedicle screws. In the future automated craniotomies should also be possible $[14,15]$, as well as complex microsurgical maneuvers. In 2002 we gained clinical and experimental experience with the 'Evolution 1' robotic system, manufactured by the former URS Inc., Schwerin, Germany [11]. Besides the fact that the system is not on the market anymore, this device did not seem to be suitable for routine clinical use. Consequently, to benefit from the potential advantages of a robotic system alternative solutions had to be found. In 2003 the robotic system 'A73' was introduced by the ENT Department of the University of Erlangen, Germany, as a system for endoscopic paranasal sinus surgery. In cooperation with the Department of Neurosurgery, University of Erlangen, Medical Intelligence Inc., Schwabmünchen, Germany, and CAS-Innovations Inc., Erlangen, Germany, an advanced set-up including a connection to the Mayfield head clamp was created. With this article we would like to introduce the new system to the field of neurosurgery and report on a preliminary assessment of application accuracy. Its potential neurosurgical applications are discussed and, based on experiences with the 'Evolution 1', technical advancements are outlined.

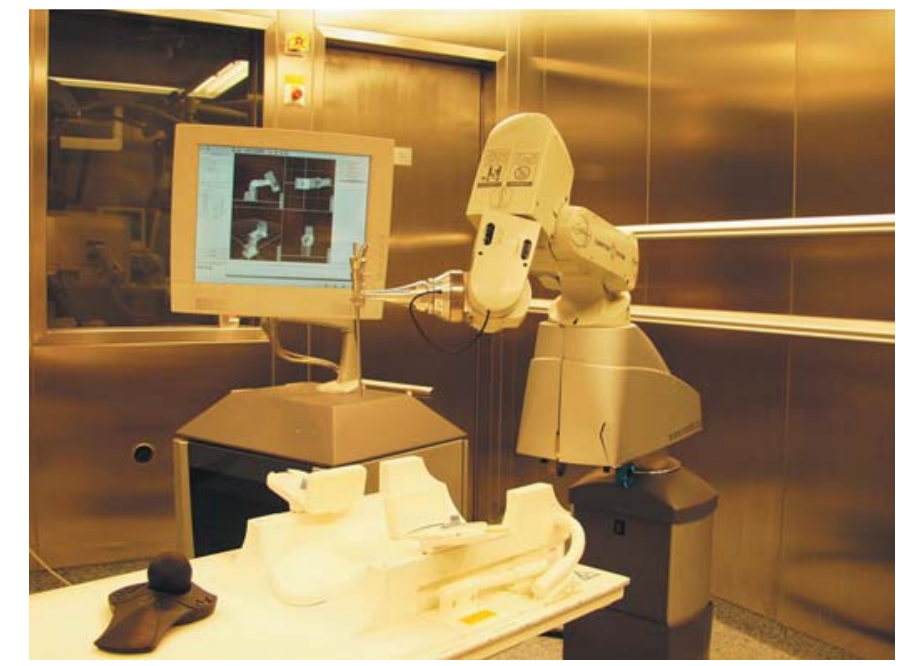

Fig. 1. Robot 'A73' with control element.

\section{Material and Methods}

\section{Robotic Setup}

The basis of the whole system is the industrial robot RV1a, Mitsubishi Electric, Tokyo, Japan, with the control element CR1-571 (fig. 1). To expand the capacity of this control element a robot-control proxy-server was integrated that coordinates not only the planning and navigational software but also the process of patient registration and various surgical instruments. Furthermore, for telemanipulatory purposes this server facilitates robot-control by a 6-D joystick (SpaceBall ${ }^{\odot}$, 3Dconnexion Inc., Germany). The robot is placed on a pedestal and can be fixed to the operating table by two mechanical arms. The working level of the robot can be adjusted to the level of the operating table by a hydraulic arm. The tip of the working arm allows attachment of different instruments. The robot itself has 6 degrees of freedom, a height of $90 \mathrm{~cm}$ and weighs $19 \mathrm{~kg}$. The maximum payload on its tool tip is $1.5 \mathrm{~kg}$, the reach is $418 \mathrm{~mm}$. With speeds up to $2,200 \mathrm{~mm} / \mathrm{s}$ positioning maneuvers can be theoretically executed with an accuracy of \pm 0.02 $\mathrm{mm}$. The system has a force-torque sensor (Schunk Inc., Germany) that can be switched on or off in the robot's control program. This sensor measures encountered resistances and applied forces, and enables the robot to respond to them with certain sensitivity. Furthermore, in a specific mode all axes of the robot are controlled by the force-torque sensor, thus allowing the manual positioning of the arms of the robot. The robotic unit possesses dedicated navigational control software; for planning and performance of surgery a specific control software was developed. For safety reasons and special applications, an optic frameless stereotactic system was integrated, the commercially available CAPPA station (CASInnovations Inc., Erlangen, Germany). Software components of both the robotic system and the external redundant navigational system CAPPA were combined to one user interface. Thus, a direct and active interference with the robotic action is possible if deviation from certain safety parameters is detected by the navigational unit. A summary of important technical data of the A73 and the Evolution 1 system is shown in table 1. 
Table 1. Technical data as provided by the manufacturers

\begin{tabular}{lcc}
\hline & Evolution 1 & A73 \\
\hline Maximum speed, mm/s & 2 & 2,200 \\
Mechanical accuracy, $\mu \mathrm{m}$ & 20 & 20 \\
Weight, kg & 300 & 19 \\
Degrees of freedom & 6 & 6 \\
Maximum payload, kg & 2.5 & 1.5 \\
Reach, mm & 160 & 418 \\
\hline
\end{tabular}

\section{Accuracy Study}

For a preliminary evaluation of clinical applicability and accuracy, we performed telemanipulatory tasks as well as fully automated ones on cadaveric heads and an individualized phantom head. For this purpose the speed of the system was adjusted to 10 $\mathrm{mm} / \mathrm{s}$. 20 CT markers were located along 3 different orthogonal sections (sagittal, axial and coronal) allowing three-dimensional analyses (fig. 2, 3, 4B, C). After CT imaging and $3 \mathrm{D}$ reconstruction, coordinates of all CT marker center points were defined in the planning software with corresponding $x-y-z$ values. All markers were measured 3 times with 10 measurements per series.

Two sources of robotic inaccuracy were evaluated: (1) the robotic reproducibility error (hardware), the combined error of robotic inaccuracy under OR scenario and inaccuracy of the navigation system, and (2) the overall error.

The reproducibility error was tested by telemanipulatory means. The robot was registered and referenced to the phantom model. The center point of a CT marker was marked by locating the tracked robot tool tip exactly into it. This point's coordinates were saved and the robot was then moved at least $20 \mathrm{~cm}$ away from the CT marker with translational and rotational movements. Afterwards the robot was told to go back to the previously marked point in a fully automated fashion without retracing the previous path. All movements are tracked by the navigation system and offsets are calculated according to deviations of the $x-y-z$ coordinates, respectively. This error reflects the actual accuracy of the robot itself and the accuracy of the navigation system. The reproducibility error was measured with 14 of the 20 CT markers located along 3 different axes. The first axis runs along the nasal septum, representing the robotic approach for transsphenoidal surgery. This transnasal axis contains 5 CT markers and measurements were taken with the first marker at the piriform aperture and the last marker in the sphenoid sinus. The axial axis was placed in a $90^{\circ}$ angle from the sphenoid sinus upwards and consists of 4 markers. The transversal axis consists of points along the lateral skull base extending from the external auditory canal to the sphenoid sinus. 5 CT markers were evaluated along this axis.

An indirect approach was used to measure the robotic overall error. Since any offset behind the marked point would result in destruction of the CT marker and phantom, a virtual elongation of the final $2 \mathrm{~mm}$ of the navigated tool tip was defined in the robotic software. Thus, the navigated tool would stop ahead of the CT marker without destroying it. The offset vector between the actual tool tip position and the previously defined target point was calculated from $x-y-z$ coordinates after moving the robot with the tool tip to the target by telemanipulatory means (translation-

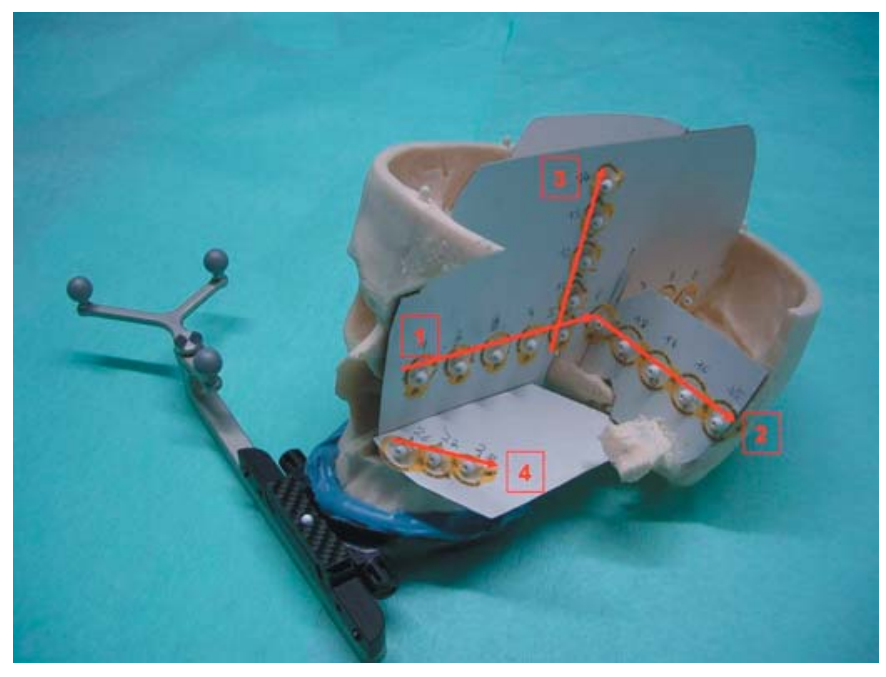

Fig. 2. Phantom model for accuracy studies with CT markers along axial, sagittal, and coronal sections. Axis 1 is referred to as transnasal, axis 2 as transversal, axis 3 as axial and axis 4 as maxillary. A further axis 'frontal' is not seen in this picture, as it is located on the other side of the skull with $3 \mathrm{CT}$ markers in the frontal paranasal sinus.

al movements only; fig. 4). The overall stereotactic error was measured as follows: $x-y-z$ coordinates of CT marker center points were preoperatively defined as target points. The robot was registered and referenced to the phantom model and the $\mathrm{x}-\mathrm{y}-\mathrm{z}$ coordinates of an arbitrary starting point were noted. After registration, the robot automatically moved its tool tip to the previously marked end point and the resulting $x-y-z$ coordinates of the final position were detected. All movements were tracked by the navigation system and offsets were calculated according to deviations in the resulting $x-y-z$ end coordinates. In addition to the transnasal, transversal and axial axes, a frontal axis with 3 markers within the frontal sinus and a maxillary axis with 3 markers within the maxillary sinus were added.

\section{Results}

Along the transnasal axis an average reproducibility error of 0.062 (range $0.03-0.11$ ) $\mathrm{mm}$ was measured. The mean error on the axial axis was 0.052 (range $0.02-0.14$ ) $\mathrm{mm}$, and 0.056 (range $0.05-0.07$ ) $\mathrm{mm}$ on the transversal axis.

The robotic overall error was measured using the above-mentioned phantom head. Accuracy data are shown in figure 5. A mean overall accuracy of 0.816 (range 0.4-1.43) mm was observed. Values for the transnasal axis were 0.63 (range $0.42-0.9$ ) $\mathrm{mm}$, for the transversal axis 0.9 (range $0.55-1.24) \mathrm{mm}$, for the axial axis 


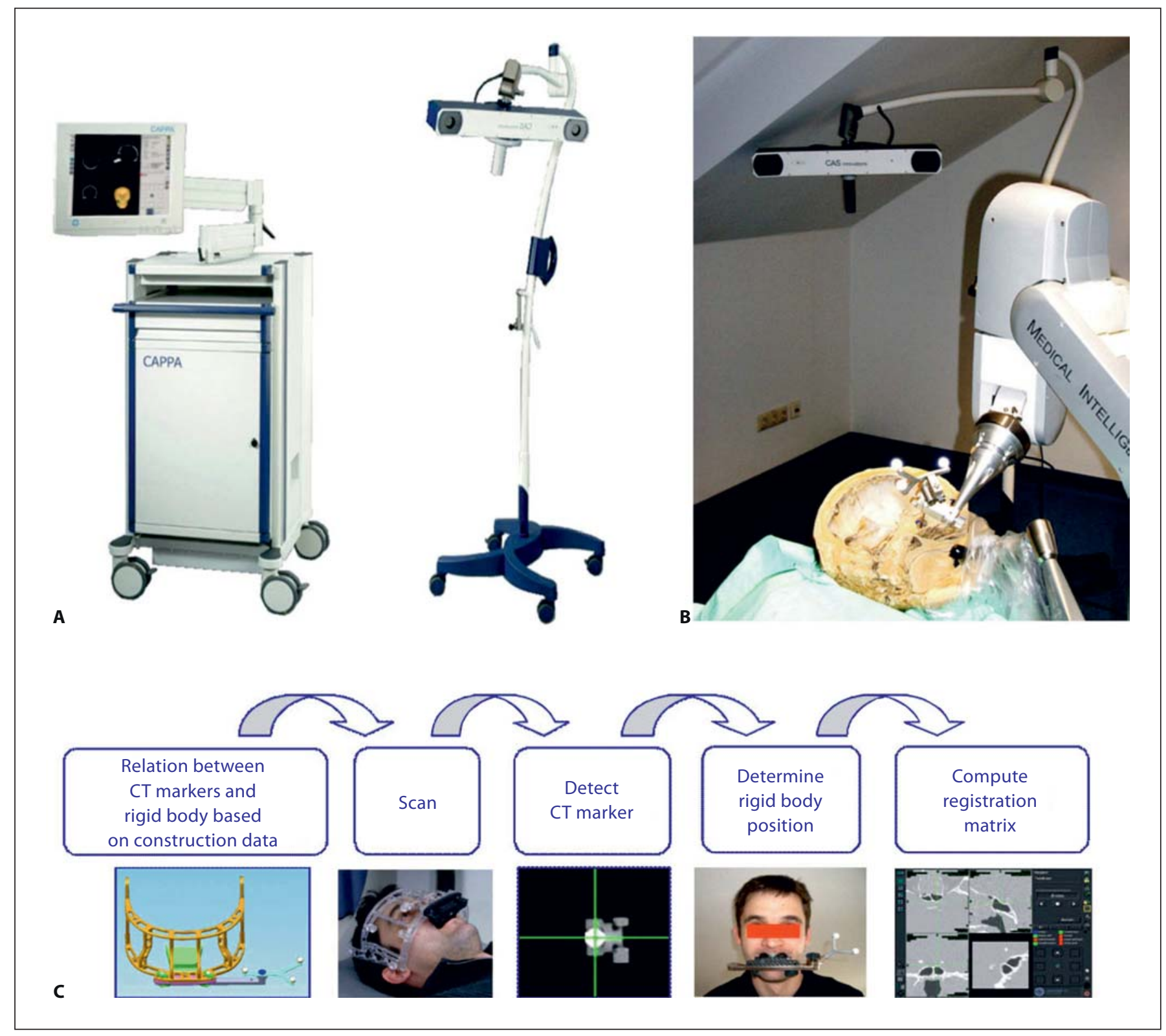

Fig. 3. Robot setup. A External and redundant navigation system. B Setup for anatomical tests. C Automated frame-based registration technique.

1.19 (range $0.9-1.43$ ) $\mathrm{mm}$, for the frontal axis 0.84 (range $0.76-0.92) \mathrm{mm}$, and for the maxillary axis 0.52 (range $0.4-0.7) \mathrm{mm}$.

The A73 system allows combination of frameless stereotaxy, robotics, and endoscopy. Fully automated procedures are possible, as well as telemanipulatory tasks. The whole system could be operated safely and stably. With regard to its size, weight and maneuverability, it is suitable for routine use in the operating theatre. The newly developed robot control software is user-friendly and makes the robot extensively programmable and flexible in its possible applications. All safety features worked reliably, no potential hazardous situations were observed. The real-time integration of frameless stereotactic data was possible and added additional safety features. 


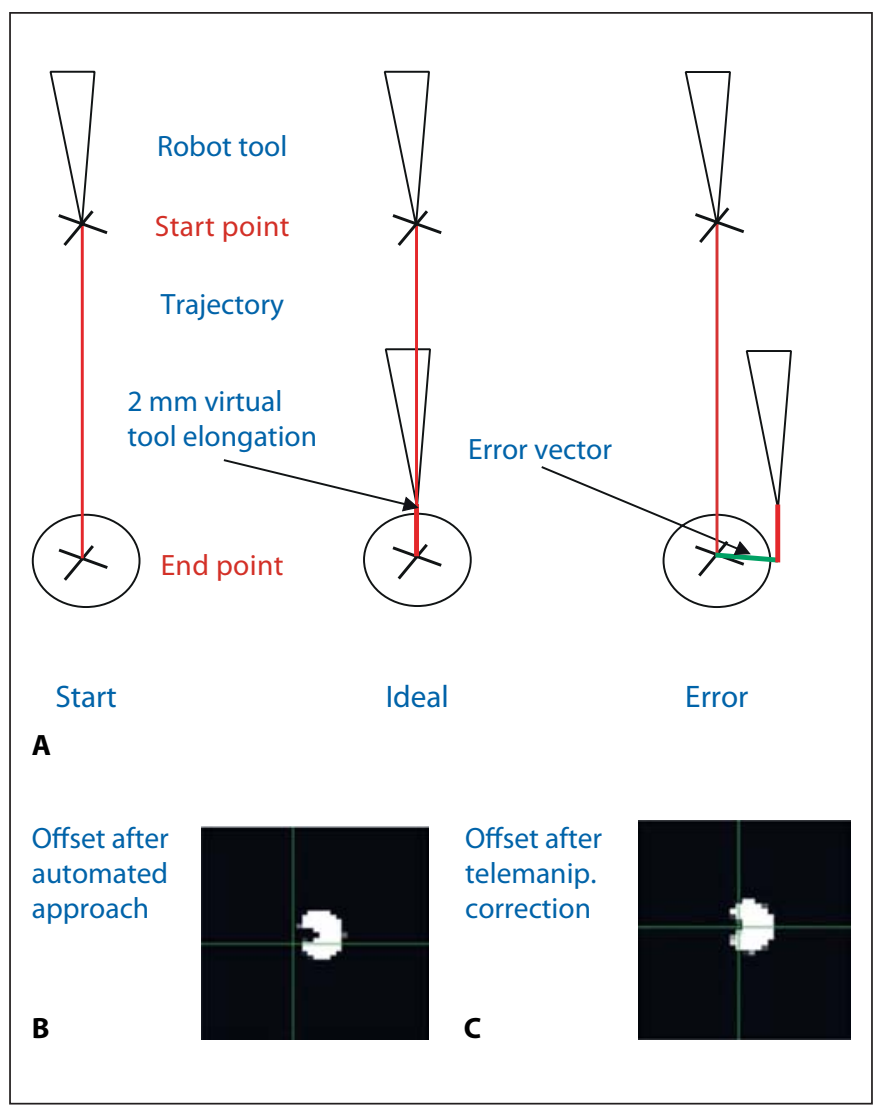

Fig. 4. Measuring overall stereotactic errors by virtual tool tip elongation.

\section{Discussion}

When working with robotic systems in the operating room, two accuracies have to be noted. The reproducibility error represents the reliability of the system when maneuvering within known and previously driven paths. For example, when the robot moves out of the surgical field along the previous entry path, the surgeon has to be sure that the tool does not damage tissue along the path due to inaccurate robot movement or tool deflections. Therefore, the reproducibility error was quantified to allow the surgeon to rely on accurate robotic movements.

The system's overall error reflects the accuracy when operating in an unknown area. It represents the real-life operating room situation, when the robot moves to a certain spot within the surgical field specified by the surgeon and performs a certain task, e.g. drilling holes. The overall accuracy therefore comprises all possible error sources that may affect the robotic action. Especially the transformation error from CT imaging to the corresponding point within the patient's head has to be noted. In this accuracy test, the surgeon marks the robot's path and end point virtually by means of CT imaging and 3D reconstructions. The error value of $0.816 \mathrm{~mm}$ represents the absolute accuracy that is accomplished after referencing the robot to the patient and initiating its fully automated movement to a spot along the frontal skull base.
Fig. 5. Error assessment with fully automated robotic actions.

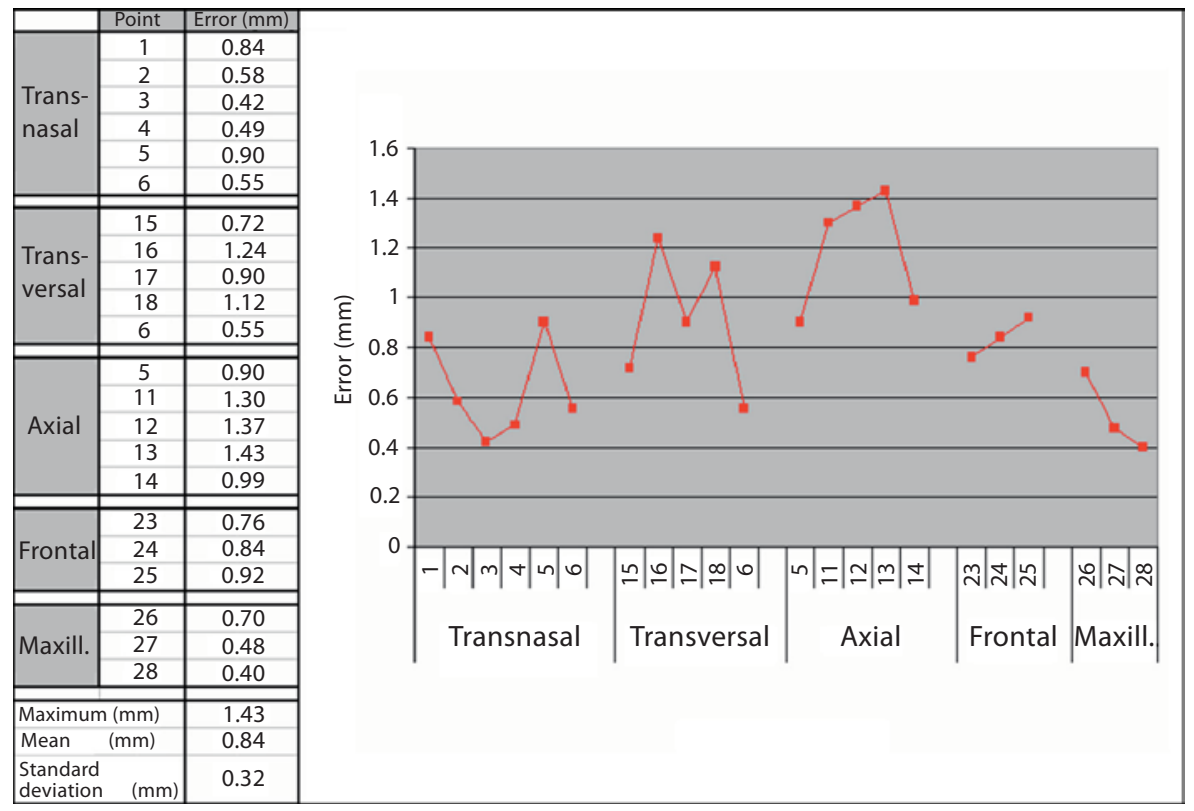

Stereotact Funct Neurosurg 2007;85:249-255 
In two previous studies we evaluated the 'Evolution 1' robotic system (formerly URS Inc., Schwerin, Germany) clinically and experimentally. This was a mobile system with 6 degrees of freedom based on a Stewart platform (hexapod) and an adapted seventh axis ( $\mathrm{z}$ axis). As there was no possibility of direct data flow between the robot and the neuronavigation system, only telemanipulatory maneuvers were performed. In a clinical study we adapted the system for endoscope-assisted transsphenoidal skull base surgery [11]. Two patients suffering from large hormonally inactive pituitary adenomas were operated with the endoscope held by the robot and tracked by the navigation system (StealthStation, Medtronic Inc., USA). Robotic assistance allowed the simultaneous use of two additional instruments under endoscopic view. Thus, drilling, suctioning and microsurgical tumor removal were feasible. From this point of view, the results were encouraging and demonstrated the potential benefit of a robotic system. To broaden the possible fields of application, we evaluated the suitability of this system in an experimental study for passive robotic-assisted insertion of pedicle screws. We utilized two human specimens and drilled the pedicles from T2 to S1 under navigational guidance using a guide tube that was positioned with the robot. After that metal rods were inserted manually. By this, pedicle screws could be inserted with a positional error of below $2 \mathrm{~mm}$ and an orientation error of below $4^{\circ}$.

In both studies the system was found to be very cumbersome, and its use very time-consuming. In placing pedicle screws the robot hindered access to the patient, therefore using the device in the operating room for this purpose seemed impossible. There was no user-friendly interface and the lack of an integrated navigation system with the possibility of direct data flow between the frameless stereotactic system and the robot's control software meant a serious limitation to the potential advantages of the system. The new system is significantly lighter and much easier to handle. The use of different surgical instruments is simple. One of the biggest advantages in comparison to the 'Evolution 1' is the integrated navigation software. By this, physical space can be referenced to image space allowing direct access of the robot's control software to the stereotactic data. Thereby, control of telemanipulatory maneuvers as well as programming of fully automated tasks is possible without the use of a separate stereotactic system requiring additional monitoring and implying potential own error sources. On the other hand, implementation of redundant frameless stereotactic control added a further safety feature and possibly makes the system more flexible for special purposes. With these features, the adaptation of the system for an automated robotic approach to minimally invasive transsphenoidal skull base surgery is already possible [16]. On cadaveric heads, fully automated sphenoidotomy was successful as well as telesurgical sphenoidectomy under endoscopic view - tasks far beyond those accomplished with systems like the 'Evolution 1'.

To minimize dural lesions in supratentorial craniotomies, the use of robot-assisted craniotomy system is suggested [17]. The system described here meets the technical requirements for performing an automated craniotomy and will be adapted to this procedure. Another consideration is the use of the system for brain biopsies. According to our experience with the system so far, installation of the burr hole as well as guidance of the biopsy needle are practicable; the latter both fully automated and telesurgical.

Several studies on misplacement of pedicle screws in spine surgery showed misplacement rates of more than $20 \%[18,19]$. By using a navigation system, these rates could be reduced, but still remain considerable [20, 21]. In our study there was some evidence that the misplacement rate might be reduced by using a robotic system. Due to the above-mentioned limitations, the system's high mechanical accuracy could not result in any relevant benefit for the surgeon or the patient. The handier new robot should not hamper the surgical procedure and the integrated navigational capacities facilitate preoperative planning and programming of the robotic actions. Therefore, based on our existing experience another study concerning the feasibility of robot-assisted insertion of pedicle screws and its influence on misplacement rates is planned.

\section{Conclusion}

The system A73 is suitable for the use in the neurosurgical operating room. With its user-friendly interface and reliable safety features, its high application accuracy and flexibility, it is a versatile robotic platform for various neurosurgical applications. An experimental automated approach for transsphenoidal skull base surgery has already been established [16] and adaptations for other uses will be realized. The question if the patient and/or the surgeon really benefit from the application of robotic systems is still to be answered. 


\section{References}

1 Howe RD, Matsuoka Y: Robotics for surgery. Annu Rev Biomed Eng 1999;1:211-240.

2 Nathoo N, Cavusoglu MC, Vogelbaum MA, Barnett GH: In touch with robotics: neurosurgery for the future. Neurosurgery 2005; 56:421-433.

3 Bargar WL, Bauer A, Borner M: Primary and revision total hip replacement using the Robodoc system. Clin Orthop 1998;354:82-91.

4 Bowersox JC, Cornum RL: Remote operative urology using a surgical telemanipulatory system: preliminary observations. Urology 1998;52:17-22.

5 Harris SJ, Arambula-Cosio F, Mei Q, Hibberd RD, Davies BL, Wickham JE, Nathan MS, Kundu B: The Prorobot: an active robot for prostate resection. Proc Inst Mech Eng [H] 1997;211:317-325.

6 Garcia-Ruiz A, Gagner M, Miller JH, Steiner CP, Hahn JF: Manual versus robotically assisted laparoscopic surgery in the performance of basic manipulation and suturing tasks. Arch Surg 1998;133:957-961.

7 Carpentier A, Loiumel D, Aupacie B, Reliand $\mathrm{J}$ : Computer-assisted cardiac surgery. Lancet 1999;353:379-380.

8 Federspil PA, Plinkert PK: Robotic surgery in otorhinolaryngology. Otolaryngol Pol 2004;58:237-242.

9 Korb W, Engel D, Boesecke R, Eggers G, Kotrikova B, Marmulla R, Raczkowsky J, Worn H, Muehling J, Hassfeld S: Development and first patient trial of a surgical robot for complex trajectory milling. Comput Aided Surg 2003;8:247-256.
10 Zimmermann M, Krishnan R, Raabe A, Seifert V: Robot-assisted navigated neuroendoscopy. Neurosurgery 2002;51:1446-1451.

11 Nimsky C, Rachinger J, Iro H, Fahlbusch R: Adaptation of a hexapod-based robotic system for extended endoscope-assisted transsphenoidal skull base surgery. Minim Invas Neurosurg 2004;47:41-46.

12 Goto T, Hongo K, Kakizawa Y, Muraoka H Miyairi Y, Tanaka Y, Kobayashi S: Clinical application of robotic telemanipulation system in neurosurgery. Case report. J Neurosurg 2003;99:1082-1084.

13 Varma TR, Eldridge PR, Forster A, Fox S, Fletcher N, Steiger M, Littlechild P, Byrne P, Sinnott A, Tyler K, Flintham S: Use of the NeuroMate stereotactic robot in a frameless mode for movement disorder surgery. Stereotact Funct Neurosurg 2003;80:132-135.

14 Eggers G, Wirtz C, Korb W, Engel D, Schorr O, Kotrikova B, Raczkowsky J, Worn $\mathrm{H}$, Muehling J, Hassfeld S, Marmulla R: Robot assisted craniotomy. Minim Invas Neurosurg 2005;48:154-158.

15 Engelhardt M, Bast P, Jeblink N, Lauer W, Popovic A, Eufinger H, Scholz M, Christmann A, Harders A, Radermacher K, Schmieder K: Analysis of surgical management of calvarial tumors and first results of a newly designed robotic trepanation system. Minim Invas Neurosurg 2006;49:98103.
16 Bumm K, Wurm J, Rachinger J, Dannenmann T, Bohr C, Fahlbusch R, Iro H, Nimsky C: An automated robotic approach with redundant navigation for minimal invasive extended transsphenoidal skull base surgery. Minim Invas Neurosurg 2005;48:159-164.

17 Engelhardt M, Uhlenbruch S, Christmann A, Miede C, Eufinger H, Scholz M, Harders A, Schmieder K: Accidental dural tears occurring during supratentorial craniotomy a prospective analysis of predisposing factors in 100 patients. Zentralbl Neurochir 2005;66:70-74

18 Vaccaro AR, Rizzolo SJ, Balderston RA, Allardyce TJ, Garfin SR, Dolinskas C, An HS: Placement of pedicle screws in the thoracic spine. Part II: an anatomical and radiographic assessment. J Bone Joint Surg Am 1995;77:1200-1206.

19 Jerosch J, Malms J, Castro WH, Wagner R, Wiesner L: Accuracy of pedicle screws following instrumented dorsal fusion of the lumbar spine (in German). Z Orthop Ihre Grenzgeb 1992;130:479-483.

20 Laine T, Lund T, Ylikowski M, Lohikoski J, Schlenzka D: Accuracy of pedicle screw insertion with and without computer assistance: a randomised controlled clinical study in 100 consecutive patients. Eur Spine J 2000; 9:235-240.

21 Amiot LP, Lang K, Putzier M, Zippel H, Labelle $\mathrm{H}$ : Comparative results between conventional and computer-assisted pedicle screw installation in the thoracic, lumbar, and sacral spine. Spine 2000;25:606-614. 